\title{
Fundamentos Para La Elaboración De Manuscritos Científicos En Trauma Y Cuidado Agudo De Emergencias (Parte 3): Como Seleccionar La Mejor Opción Para Publicar Su Trabajo: Más Allá Del Factor De Impacto
}

\author{
${ }^{1}$ María A Oviedo, ${ }^{2}$ Angel F León, ${ }^{3}$ Jorge H Montenegro, ${ }^{4}$ Raúl A Echeverri, ${ }^{5}$ Andrés M Rubiano
}

\begin{abstract}
RESÚMEN
El número de revistas científicas aumenta cada año generando así una gran variedad de información y múltiples opciones para enviar artículos producto de investigaciones. Existen índices bibliométricos que permiten ayudar a evaluar revistas para definir que tan frecuente se publican en ellas artículos de interés para generar otras publicaciones a partir de estos artículos. Medidas como el factor de impacto o el factor $\mathrm{H}$, actualmente son usadas a nivel global para identificar revistas y parametrizar autores de alta citación. Actualmente las revistas impresas estan siendo reemplazadas por las revistas electrónicas, y esta tendencia ha favorecido la proliferación de revistas o editoriales predatorias, que encuentran en la necesidad de visibilidad académica de muchos autores una oportunidad para generar un flujo importante de dinero, cobrando por la publicación de los artículos. Debido a esto, seleccionar la revista a la cual se envian los artículos es una importante tarea que debe ser realizada con precaución y bajo la supervisión de los mentores de investigación. Existen actualmente sitios web que nos ayudan a identificar revistas de riesgo o editoriales de riesgo para no caer en este tipo de situaciones en donde nuestro artículo puede ser considerado una publicación de dudosa calidad al no pasar por un proceso extricto de revisión por pares. Revistas generales reconocidas de editoriales de calidad o revistas especializadas de asociaciones científicas de alta calidad académica deben ser la primera opción a considerar independiente de los índices bibliométricos.
\end{abstract}

Palabras Clave: Artículos científicos, Factor de impacto, Índice $\mathrm{H}$, Revistas predatorias.

\section{ABSTRACT}

The number of scientific journals increases every year, generating a great variety of information and multiple opportunities for articles submission. There are bibliometric indexes that support journal evaluations in order to define how often they publish useful and high quality academic articles. Measures, such as

\footnotetext{
${ }^{1,2}$ Student, ${ }^{3,4}$ Research Associate, ${ }^{5}$ Professor

1,2Medical School, Neuroscience Institute, El Bosque University Bogotá, Colombia

${ }^{3,4}$ Clinical Research Group, MEDITECH Foundation, Neiva Huila, Colombia

${ }^{5}$ Department of Neuroscience, El Bosque University/Medical \& Research Director, MEDITECH Foundation, Bogotá, Colombia

Correspondant Author: Andrés M Rubiano, El Bosque University/Meditech Foundation, Av. Cra 9 \#130A-2, Bogotá Colombia, Phone: +57-300-6154775, e-mail: andresrubiano@ aol.com
}

the impact factor or the $\mathrm{H}$ factor, are currently used globally to identify quality journals and high-citation authors. Currently, printed journals are being replaced by electronic journals, and this tendency has favored the proliferation of predatory journals and editorials. Many authors desperate looking for academic visibility or easy publication process pay the price for publication in these low quality journals. Because of this, selecting the journal for article submission is an important task that must be done with caution and under the supervision of research mentors. Actually there are websites that help us identify predatory journals or publishers avoiding to fall into such situations where our article may be considered a dubious quality publication by not going through a sound or regular peer review process. Recognized high quality general journals of sound editorial houses or specialized journals of high academic quality associations should be the first option of submission independent of the bibliometric indexes.

Keywords: H index, Impact factor, Predatory journals, Scientific articles.

How to cite this article: Oviedo MA, León AF, Montenegro JH, Echeverri RA, Rubiano AM. Fundamentos Para La Elaboración De Manuscritos Científicos En Trauma Y Cuidado Agudo De Emergencias (Parte 3): Como Seleccionar La Mejor Opción Para Publicar Su Trabajo: Más Allá Del Factor De Impacto. Panam J Trauma Crit Care Emerg Surg 2017;6(1):35-43.

Source of support: Nil

Conflict of interest: None

\section{INTRODUCCIÓN}

El avance actual de las fuentes de información en salud ha permito ampliar las posibilidades al personal de salud para acceder a artículos y a la vez para publicar documentos científicos. Este crecimiento exorbitante incrementa a la vez las posibilidades de sesgo por la amplia gama de fuentes para consultar y publicar, dentro de las cuales no siempre existe un proceso exhaustivo de revisión académica de alta calidad. ${ }^{1}$

El número de revistas científicas aumenta cada año generando así una gran variedad de información que debe ser apropiadamente evaluada con la finalidad de verificar si la investigación presentada se ha llevado a cabo de forma correcta o si no existen elementos que induzcan fallas en la ética de publicación. ${ }^{1,2}$

Anualmente se publican más de 1,4 millones de artículos cientificos y se calcula que el número de artículos publicados mensualmente bajo un pobre sistema de revisión puede sobrepasar los 16.000 documentos. Existen índices bibliométricos, los cuales son herramientas que de manera 
cuantitativa tratan de medir la calidad de la información de una revista científica, siendo una de las mas conocidas el factor de impacto. La alta calidad en las publicaciones científicas, incrementa el interés de la comunidad académica y de los investigadores para publicar y a su vez promueve que se generen estudios que realmente aporten al avance de la ciencia. En este marco debe promoverse la interacción entre investigadores junior e investigadores senior que a su vez ejercen como mentores en la elaboración de artículos científicos. Durante el desarrollo de estos procesos de investigación, los métodos o sistemas de medición de calidad de las revistas indexadas se convierten en elementos esenciales para definir a donde se envian los artículos resultado de la investigación. ${ }^{2}$

En el contexto latinoamericano, sólo recientemente se han considerado los índices bibliométricos cómo elementos de importancia para definir a donde se envian los artículos. Igualmente se ha generado recientemente un riesgo de acceder a revistas denominadas pseudorevistas o revistas de editoriales predatorias, las cuales han aprovechado el interés de científicos para publicar y a través de una amplia campaña de propaganda via internet, generan espacios motivados netamente por intereses económicos, con muy poca o ninguna revisión académica de los manuscritos recibidos.,

En este artículo, pretendemos revisar algunos conceptos fundamentales a tener en cuenta a la hora de considerar una revista para enviar artículos de resultados de proyectos de investigación que aporten al avance del campo del trauma y el cuidado agudo de emergencias.

\section{Indices Bibliométricos}

\section{Factor de impacto}

El Factor de Impacto es una herramienta elaborada anualmente por el Instituto para la Información Científica, que tiene como fin medir la influencia, la importancia y el nivel de investigación de las revistas indexadas en las bases de citaciones más importantes. Una de estas es el denominado Reporte de Citaciones de Revistas (Journal Citation Reports). 5,6 El Factor de impacto consiste en la realización de un cálculo matemático que tiene en cuenta el número de citaciones realizadas de una revista a lo largo del año para la construcción de nuevos artículos en otras revistas. A mayor número de citaciones hechas en otros artículos acerca de estudios publicados en la revista, mayor será su factor de impacto. Esta medición ha sido criticada ya que el factor de revistas muy especializadas es menor que el de revistas generales y las revistas no indexadas igualmente pueden presentar un factor mucho menor. Adicionalmente es importante tener en cuenta que no todos los articulos se consideran citables. Se tienen en cuenta para estas mediciones de impacto unicamente estudios tipo meta analisis, revisiones sistemáticas, estudios clinicos aleatorizados, estudios de cohortes, etc. No se incluyen reportes de casos, series de casos o cartas a los editores. Por este motivo, revistas que publiquen unicamente artículos con este tipo de estudios, tendrán un mayor factor de impacto. Revistas que publiquen reportes de casos, revisiones nó sistemáticas y series de casos tendrán un factor de impacto más bajo. ${ }^{6-8}$

La importancia del Factor de Impacto radica en la posibilidad que tienen los autores de los diferentes estudios para conocer qué revista tiene una mayor difusión a nivel general o en un determinado campo y de esta manera escoger con mejores argumentos la más idónea para publicar sus investigaciones. La importancia de una investigación científica puede medirse según el impacto que tiene en la comunidad académica, así como su importancia y relevancia en la creación de nuevo conocimiento. Este impacto de alguna $\mathrm{u}$ otra forma puede ser dado de un modo cualitativo que puede determinar que tanta difusión tiene un artículo, y por tanto que tanta interacción con lectores generarán sus resultados. En el contexto internacional el análisis de las citas es fundamental en la evaluación de la literatura y esto se ha intensificado en los últimos 4 años por medio de la bibliometría de la investigación científica, de la que hace parte el factor de impacto.?

El Factor de impacto, se define por el Instituto para la Información Científica en la relación de dos elementos, el primero el denominador en el que se incluye en número total de artículos publicados en los dos últimos años en una revista en particular que pueden ser citados. El segundo el numerador en el que se incluyen todas las citaciones de artículos publicados en los dos últimos años durante el año en curso. Para la aplicación del cálculo del factor de impacto, se utiliza la fórmula de la Figura 1. La letra $\mathrm{X}$ identifica a la revista y la letra $\mathrm{Y}$ el año en el que se realizó el cálculo: ${ }^{3}$

Por ejemplo, para el cálculo del Factor de impacto del Acta Médica Colombiana, para 2014 se tiene el siguiente cálculo (Tabla 1):

\# de citaciones en $\mathrm{Y}$ de articulos publicados en $\mathrm{X}$ entre (Y-1) a (Y-2) $\mathrm{F} \mid \mathrm{X}=\frac{}{\# \text { de articulos citables publicados en } \mathrm{X} \text { entre }(\mathrm{Y}-1) \text { a }(\mathrm{Y}-2)}$

Figura 1: Fórmula de cálculo del factor de impacto

Tabla 1: Cálculo factor de impacto para el Acta Médica Colombiana en 2014

\begin{tabular}{|c|c|c|c|c|c|c|c|}
\hline \multicolumn{4}{|c|}{ Citas en 2014 para } & \multicolumn{3}{|c|}{ Artículos publicados en } & \multirow{2}{*}{$\begin{array}{l}\text { Factor de } \\
\text { impacto }\end{array}$} \\
\hline Todos los años & 2013 & 2012 & $2013+2012$ & 2013 & 2012 & $2013+2012$ & \\
\hline 108 & 8 & 4 & 12 & 48 & 40 & 88 & 0.13 \\
\hline
\end{tabular}




\section{Factor $\mathrm{H}$}

El Factor $\mathrm{H}$ también conocido como Índice $\mathrm{H}$, descrito por Jorge Hirsch, es una herramienta bibliométrica recientemente utilizada, que funciona de la misma manera que el factor de impacto como un sistema de medida de la calidad profesional de un autor de una revista, de una universidad o de un país, siendo una herramienta que ha permitido mejorar la manera en que se evalúan los investigadores.

El índice $H$ es un número que caracteriza a un investigador el cual de $X$ número de artículos publicados, tiene por lo menos $Y$ número de citaciones por artículo, donde el valor de X y $Y$ debe ser el mismo. Este valor se considera el Índice $H$, es decir, si un autor tiene 50 publicaciones, pero sólo 20 han sido referencias, y sólo un total de 15 han sido citados 15 veces o más, su factor $H$ es 15 . En él Gráfico 1, se muestra el ejemplo del cálculo del Factor $H$, para uno de los autores de este artículo, que tiene en total 356 citaciones, 315 de estas desde el 2012, pero donde sólo 10 de estas 315 citaciones han sido citadas 10 veces o más. Por esto el Factor H ó índice H de este autor es de 10 para 2016. Una subdivisión de este índice es el índice i10, en el cual se preestablece el valor de H como 10. En el ejemplo de este autor el índice i10 para 2017, presenta 11 referencias que se han citado 10 veces o más para 2017. 9,10

Este índice bibliométrico es una herramienta que se caracteriza por tener una única variable en estudio, dicha variable es el número de artículos ordenados por frecuencia de citas recibidas, lo que de manera directa produce una ventaja fundamental en la productividad global y sostenida del investigador, que lleva a un incremento del índice bibliográfico. Adicionalmente se destaca por ser una herramienta de gran utilidad y estabilidad, así como el incremento que tiene con el paso de la vida profesional científica. Actualmente este sistema es el utilizado por Google Académico para clasificar las referencias y

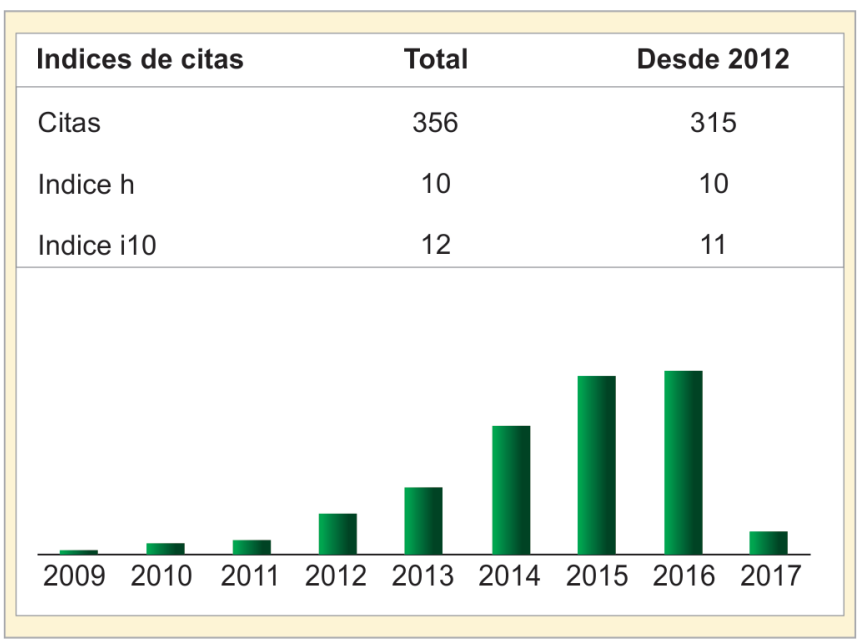

Gráfico 1: Ejemplo para calcular el Factor $\mathrm{H}$ citaciones de los autores institucionales inscritos en esta plataforma.

El factor $H$ permite de alguna manera valorar aquellos estudios que son considerablemente buenos y que son avalados por un número importante de citaciones, pero que han sido publicados en revistas de bajo factor de impacto. La importancia de esto radica en que autores que deciden publicar en revistas de poco impacto, pueden ser comparados cuantitativamente con autores que publican artículos en revistas de alto impacto. Por lo tanto, este es un índice que puede ser utilizado para medición de impacto en revistas nó indexadas o que tienen bajo impacto medido por otros índices., ${ }^{9,10}$

\section{Criterios Para Seleccionar Una Revista}

Si se compara años atrás, las publicaciones en revistas se obtenían únicamente por medio físico, hoy esto ha cambiado y gracias a los avances tecnológicos y a las plataformas en línea para investigar, las revistas se encuentran además en formato digital lo cual ha permitido más vías de acceso que han hecho que publicar genere una amplia gama de posibilidades con diferentes ofertas y oportunidades en los procesos de investigación. Estas revistas fisicas o digitales se convertirán en una fuente de búsqueda importante entre sus consultores. Una revista tiene que satisfacer las necesidades tanto del autor como del lector y para esto debe cumplir con funciones como: un adecuado registro, certificación que la investigación se haya llevado a cabo de forma correcta, difusión al público adecuado, acceso a los diferentes tipos de lectores, permanencia de un registro público permanente, y acceso a medios electrónicos. Respecto a publicaciones de artículos y ante tanta oferta que constantemente se recibe, y como anteriormente se mencionó, con la revolución del internet, hay información de fácil acceso que precisamente genera riesgo de que el investigador pueda ser engañado. Hay que saber elegir las revistas donde se quiere publicar y de esta manera evitar trampas y lograr un trabajo con mayor eficacia para lo cual se debe tener en cuenta si la revista está indexada, comprobar la base de datos, nombre del editor, dirección registrada, entre otros. Al momento de escribir un artículo, es importante saber a que revista se enviará; hay que tener en cuenta que sea reconocida y/ó especializada en el tema que hace parte del proyecto de investigación. Generalmente la primera opción puede ser una revista de alto impacto y amplia cobertura. En caso de que el artículo no sea aprobado, se podrá enviar a una revista de menor impacto o de menor cobertura. ${ }^{6-10}$

En la Tabla 2, se encuentra la lista de las 20 primeras revistas con Factor de impacto más alto para el año 2014. Generalmente son revistas que publican investigaciones en temas generales o que publican temas especializados 
Tabla 2: Factor de impacto de las primeras 20 revistas biomédicas para 2014

\begin{tabular}{lll}
\hline Nombre de revista & \# De citaciones & Factor de \\
para 2011 & Impacto \\
\hline Ca-Cancer J Clin & 18594 & 115,840 \\
New Engl J Med & 268652 & 55,873 \\
Chemical Reviews & 137600 & 46,568 \\
Lancet & 185361 & 45,217 \\
Nature Reviews Drug & 23811 & 41,908 \\
Nature Biotechnology & 45986 & 41,514 \\
Nature & 617363 & 41,456 \\
Annual Rev Immunology & 16750 & 39,375 \\
Nature Rev Molec Cell & 35928 & 37,806 \\
Nature Rev Cancer & 39868 & 37,400 \\
Nature Rev Genetics & 29388 & 36,978 \\
Nature Materials & 64622 & 36,503 \\
Jama J American Med & 126479 & 35,289 \\
Nat Rev Immunology & 28938 & 34,985 \\
Nat Nanotech & 34387 & 34,048 \\
Science & 557558 & 33,611 \\
Chemical Society Rev & 81907 & 33,383 \\
Ann Rev Astron Astroph & 8462 & 33,346 \\
Nature Photonics & 23499 & 32,386 \\
Cell & 201108 & 32,242 \\
\hline
\end{tabular}

especialmente de artículos de investigación de ciencias básicas, que posteriormente seran muy citados en investigaciones clínicas.

La única manera de identificar la mejor calidad de una revista científica, sería la evaluación de las diferentes publicaciones por expertos calificados, algo que resultaría muy complicado; por tal razón, si bien el factor de impacto debe ser empleado, no debe utilizarse como medida única de la actividad científica y debe complementarse con otro tipo de medidas bibliometricas, en especial en zonas en las cuales existen revistas de bajo impacto con aportes importantes en la literatura científica como ocurre en España, Francia, Italia y América Latina. ${ }^{7-10}$

\section{Revistas que publican artículos científicos especializados en trauma y cuidado agudo de emergencias}

De acuerdo a la actualización hecha por el Journal Citation Reports para el 2015 (basada en citaciones de 2013 y 2014), 6 revistas indexadas, reconocidas, de amplia cobertura que podrían recibir artículos de trauma son descritas a continuación (Tabla 3$){ }^{8}$

Al momento de elegir dónde publicar, las revistas epidemiológicas o especializadas en salud pública se convierten también en una buena alternativa, siempre y cuando estén bien posicionadas. En este tipo de publicaciones el proceso de selección es muy estricto específicamente en lo referente a la metodología. En la tabla 4, se describen revistas especializadas a nivel mundial con temática relacionada a trauma y cuidado agudo de emergencias,
Tabla 3: Revistas indexadas generales de alto impacto que pueden recibir artículos de trauma o cuidado agudo de emergencias

\begin{tabular}{llll}
\hline & & $\begin{array}{l}\text { Número } \\
\text { de citas } \\
\text { anuales }\end{array}$ & $\begin{array}{l}\text { Factor de } \\
\text { impacto }\end{array}$ \\
\hline 2 & $\begin{array}{l}\text { New England Journal of } \\
\text { Medicine }\end{array}$ & 283,525 & 59.558 \\
& Lancet & 195,553 & 44.002 \\
4 & Nature & 627,846 & 38.138 \\
9 & JAMA (Journal of the American & 129,909 & 37.684 \\
11 & Medical Association) & & \\
& Science & 568,210 & 34.661 \\
16 & BMJ (British Medical Journal) & 93,118 & 19.697 \\
\hline 50 & & &
\end{tabular}

en orden alfabético y con su respectivo factor de impacto en el año 2015. ${ }^{8,9}$

\section{Pseudorevistas y Editoriales Predatorias}

La publicación y divulgación del conocimiento requiere un proceso estricto y riguroso de revisión, sin embargo se ha evidenciado desde hace varios años métodos irregulares tanto de autores como editores para mejorar de forma rápida su curriculum vitae y lograr retribución económica respectivamente; a las revistas que "facilitan" estos procesos se las conoce como "pseudorevistas" y a las editoriales que las publican como "editoriales depredadoras" ocasionando perjuicios en otras revistas de excelente reputación. ${ }^{11}$

Estas revistas han surgido como consecuencia de el auge del open access, aproximadamente después del año 2000, cuando muchas revistas entraron en la tendencia del acceso abierto, cobrando a los autores por la publicación de sus artículos. Muchas editoriales de poca calidad, especialmente provenientes de Asia, vieron la oportunidad de ganar dinero "facil" promoviendo revistas con una mínima o ninguna revisión especializada por pares, en donde prácticamente por el solo hecho de pagar, se garantiza la publicación. Este modelo se ha considerado una práctica de explotación académica, aprovechandose de la necesidad de los autores por transmitir el conocimiento y un cambio en el modelo dominante del negocio de las publicaciones, pasando de las revistas impresas a las revistas electrónicas. Muchas de estas revistas han sido objeto de seguimiento y evaluación. Uno de los portales más conocidos para identificar estas revistas y editoriales, era el del Bibliotecario Jeffrey Beall de la Universidad de Colorado, quien desafortunadamente decidió cerrar este web site en enero de 2017 (Figura 2). ${ }^{10-14}$

Este website, identificó desde el 2010, un aumento descomunal del numero de editoriales y revistas predatorias, exponiendo de manera importante ante la comunidad científica el riesgo que se puede correr a la hora de seleccionar revistas para publicar artículos científicos 
Tabla 4: Lista de revistas científicas a nivel mundial relacionadas con trauma y cuidado agudo de emergencias en el año 2015

\begin{tabular}{|c|c|c|}
\hline Nombre de la revista & País de la Editorial & Factor de Impacto \\
\hline Acta chirurgiae orthopaedicae et traumatologiae Cechoslovaca & Republica Checa & 0,38 \\
\hline Acta orthopaedica et traumatologica turcica & Turquía & 0,61 \\
\hline Acta ortopedica brasileira & Brasil & 0,15 \\
\hline Acta ortopédica mexicana & México & 0,07 \\
\hline Advances in skin \& wound care & Estados Unidos & 1,11 \\
\hline Advances in wound care & Estados Unidos & - \\
\hline African journal of traumatic stress & Uganda & - \\
\hline Annals of advances in automotive medicine & Estados Unidos & - \\
\hline Annals of burns and fire disasters & Italia & - \\
\hline Annals of maxillofacial surgery & India & - \\
\hline Archives of orthopaedic and trauma surgery & Alemania & 1,59 \\
\hline Archives of trauma research & Irán & 0,18 \\
\hline Asian journal of sports medicine & Irán & - \\
\hline Australasian Journal of Disaster and Trauma Studies & Nueva Zelanda & - \\
\hline BMC emergency medicine & Inglaterra & - \\
\hline Brain injury & Inglaterra & 1,82 \\
\hline Burns: journal of the International Society for Burn Injuries & Holanda & 1,88 \\
\hline China journal of orthopaedics and traumatology & China & - \\
\hline Chinese journal of burns & China & - \\
\hline Chinese journal of traumatology & China & - \\
\hline Chronic diseases and injuries in Canada & Canadá & 1,63 \\
\hline Clinical journal of sport medicine & Canadá & 2,26 \\
\hline Cognitive, affective \& behavioral neuroscience & Estados Unidos & 3,28 \\
\hline Craniomaxillofacial trauma \& reconstruction & Estados Unidos & - \\
\hline Current sports medicine reports & Estados Unidos & 1,60 \\
\hline Dental traumatology & Dinamarca & 1,60 \\
\hline Der Unfallchirurg & Alemania & 0,58 \\
\hline Developmental neurorehabilitation & Inglaterra & 2,05 \\
\hline Dialysis, transplantation \& burn & Turquía & - \\
\hline ED management: the monthly update on emergency department management & Estados Unidos & - \\
\hline Emergency medicine reports & Estados Unidos & - \\
\hline European journal of orthopaedic surgery \& traumatology & Francia & 0,18 \\
\hline European journal of trauma and emergency surgery & Alemania & 0,34 \\
\hline European orthopaedics and traumatology & Alemania & - \\
\hline Evidence-based spine-care journal & Alemania & 2,16 \\
\hline Foot \& ankle & Estados Unidos & 0,74 \\
\hline Foot \& ankle specialist & Estados Unidos & - \\
\hline Foot and ankle clinics & Inglaterra & 0,75 \\
\hline Foot and ankle international & Estados Unidos & 1,50 \\
\hline Global spine journal & Alemania & - \\
\hline Hand surgery: an international journal devoted to hand and upper limb & Singapur & - \\
\hline Hand Therapy & Inglaterra & 2,00 \\
\hline Injury & Inglaterra & 1,91 \\
\hline Injury prevention & Inglaterra & 1,69 \\
\hline International journal of burns and trauma & Estados Unidos & - \\
\hline International journal of injury control and safety promotion & Inglaterra & 0,70 \\
\hline International Journal of Orthopaedic and Trauma Nursing & Inglaterra & - \\
\hline International wound journal & Inglaterra & 2,59 \\
\hline $\begin{array}{l}\text { Intervention: international journal of mental health, psychosocial work, and counselling in } \\
\text { areas of armed conflict }\end{array}$ & Holanda & - \\
\hline Journal de Traumatologie du Sport & Francia & - \\
\hline Journal of aggression, maltreatment \& trauma & Estados Unidos & - \\
\hline Journal of applied biomechanics & Estados Unidos & 0,98 \\
\hline Journal of athletic training & Estados Unidos & 2,01 \\
\hline Journal of avian medicine and surgery & Estados Unidos & 0,55 \\
\hline
\end{tabular}


(Cont'd)

Nombre de la revista

Journal of brachial plexus and peripheral nerve injury

Journal of burn care and research

Journal of child \& adolescent trauma

Journal of children's orthopaedics

Journal of clinical orthopaedics and trauma

Journal of dance medicine \& science

Journal of emergencies, trauma, and shock

Journal of foot and ankle research

Journal of hand and microsurgery

Journal of injury \& violence research

Journal of loss \& trauma

Journal of maxillofacial trauma

Journal of neuroengineering and rehabilitation

Journal of neurotrauma

Journal of orthopaedic surgery and research

Journal of orthopaedic trauma

Journal of Orthopaedics, Trauma and Rehabilitation

Journal of sport rehabilitation

Journal of tissue viability

Journal of trauma \& dissociation

Journal of trauma management \& outcomes

Journal of trauma nursing

Journal of traumatic stress

Journal of wound care

Journal of wrist surgery

Journal of the Korean Association of Oral and Maxillofacial Surgeons

Knee surgery, sports traumatology, arthroscopy

Magyar traumatológia, ortopédia, kézsebészet és plasztikai sebészet

Military medicine

Minerva Ortopedica e Traumatologica

Muscles, ligaments and tendons journal

Neuropsychological rehabilitation

NeuroRehabilitation

Neurorehabilitation and neural repair

Open access journal of sports medicine

Operative Orthopädie und Traumatologie

Operative techniques in sports medicine

ORL-head and neck nursing

Orthopaedics \& Traumatology Surgery \& Research

Orthopaedics and trauma

Ortopedi och traumatologi i Finland

Ortopediia travmatologiia i protezirovanie

Ostomy/wound management

Panamerican Journal of Trauma, Critical Care \& Emergency Surgery

Physical therapy in sport: official journal of the Association of Chartered

Psychological trauma: theory, research, practice and policy

Research in sports medicine

Revista Cubana de Ortopedia y Traumatologia

Revista española de cirugía ortopédica y traumatología

Revue de Chirurgie Orthopedique et Traumatologique

Revue suisse de médicine et de traumatologie du sport

Scandinavian journal of medicine \& science in sports

Scandinavian journal of trauma, resuscitation and emergency medicine

Seminars in musculoskeletal radiology

Spinal cord

\begin{tabular}{|c|c|}
\hline País de la Editorial & Factor de Impacto \\
\hline Holanda & 0,95 \\
\hline Inglaterra & - \\
\hline Estados Unidos & 1,42 \\
\hline Estados Unidos & - \\
\hline Alemania & 1,16 \\
\hline India & - \\
\hline Estados Unidos & - \\
\hline India & - \\
\hline Estados Unidos & 1,48 \\
\hline India & - \\
\hline Irán & - \\
\hline Inglaterra & 1,03 \\
\hline Italia & - \\
\hline Inglaterra & 2,57 \\
\hline Estados Unidos & 4,37 \\
\hline Inglaterra & 1,21 \\
\hline Estados Unidos & 1,80 \\
\hline China & - \\
\hline Estados Unidos & 1,27 \\
\hline Inglaterra & 1,65 \\
\hline Inglaterra & 1,54 \\
\hline Inglaterra & 0,95 \\
\hline Estados Unidos & 0,55 \\
\hline Estados Unidos & 2,62 \\
\hline Inglaterra & 1,06 \\
\hline Inglaterra & - \\
\hline Corea (Sur) & - \\
\hline Alemania & 3,09 \\
\hline Hungría & - \\
\hline Estados Unidos & 0,77 \\
\hline Italia & - \\
\hline Italia & - \\
\hline Estados Unidos & 1,95 \\
\hline Holanda & 1,12 \\
\hline Estados Unidos & 3,98 \\
\hline Nueva Zelanda & - \\
\hline Alemania & 1,03 \\
\hline Inglaterra & 0,36 \\
\hline Estados Unidos & - \\
\hline Francia & 1,39 \\
\hline Inglaterra & 1,80 \\
\hline Finlandia & - \\
\hline Ucrania & - \\
\hline Estados Unidos & 1,12 \\
\hline India & - \\
\hline Inglaterra & 1,57 \\
\hline Estados Unidos & 2,31 \\
\hline Inglaterra & 1,70 \\
\hline Cuba & - \\
\hline España & - \\
\hline Francia & 0,55 \\
\hline Suiza & - \\
\hline Dinamarca & 2,90 \\
\hline Inglaterra & 2,31 \\
\hline Estados Unidos & 1,09 \\
\hline Inglaterra & 1,80 \\
\hline
\end{tabular}




\begin{tabular}{lll}
\hline Nombre de la revista & País de la Editorial & Factor de Impacto \\
\hline Sport Orthopädie Traumatologie & Alemania & - \\
Sports health & Estados Unidos & - \\
Sports medicine and arthroscopy review & Estados Unidos & 1,68 \\
Sportverletzung Sportschaden: Organ der Gesellschaft fur Orthopadisch- & Alemania & 0,28 \\
Traumatologische Sportmedizin & & - \\
Strategies in trauma and limb reconstruction & Italia & 3,31 \\
The bone \& joint journal & Inglaterra \\
The international journal of lower extremity wounds & Estados Unidos \\
The Journal of head trauma rehabilitation & Estados Unidos \\
The journal of spinal cord medicine & Inglaterra & 3,36 \\
The journal of the American College of Clinical Wound Specialists & Estados Unidos \\
The journal of trauma and acute care surgery & Estados Unidos \\
The Knee & Holanda & - \\
Topics in spinal cord injury rehabilitation & Estados Unidos \\
Traffic injury prevention & Inglaterra \\
Trauma monthly & Irán \\
Trauma und Berufskrankheit & Alemania \\
Trauma, violence \& abuse & Inglaterra \\
Traumatology & Inglaterra \\
Turkish journal of trauma \& emergency surgery (Ulusal travma ve acil cerrahi dergisi) & Turquía \\
Wounds: A compendium of clinical research and practice & Estados Unidos & - \\
\hline
\end{tabular}

\section{BEALL'S LIST of (0) PREDATORY
ace
PUBLISHERS}

\section{7}

Figura 2: Website de Revistas y Editoriales Predatorias del Bibliotecario Jeffrey Beall. Disponible en https://web.archive.org/ web/20170111172023/https://scholarlyoa.com/2017/01/03/beallslist-of-predatory-publishers-2017/

(Tabla 5). Adicionalmente se han identificado sitios web que suplantan plataformas editoriales de revistas reconocidas para para cobrar dinero por la publicación. ${ }^{15,16}$

En América Latina el programa SciELO (Scientific electronic library online) adoptó desde 1998 el acceso abierto convirtiéndose en la región con mayor número de revistas evaluadas por pares disponibles a texto completo seguido por Portugal, España y África del Sur. El costo
Tabla 5: Editoriales y revistas predatorias desde 2011 a 2017 según el seguimiento de la Lista de Beall

\begin{tabular}{|c|c|c|c|}
\hline$A \tilde{N} O$ & $\begin{array}{l}\text { Editoriales } \\
\text { Predatorias }\end{array}$ & $\begin{array}{l}\text { Revistas } \\
\text { Independientes } \\
\text { Predatorias }\end{array}$ & $\begin{array}{l}\text { Revistas Suplantadas } \\
\text { o Usurpadas en } \\
\text { Internet }\end{array}$ \\
\hline 2011 & 18 & - & - \\
\hline 2012 & 23 & - & - \\
\hline 2013 & 225 & 126 & - \\
\hline 2014 & 477 & 303 & - \\
\hline 2015 & 693 & 507 & 30 \\
\hline 2016 & 923 & 882 & 101 \\
\hline 2017 & 1155 & 1294 & 115 \\
\hline
\end{tabular}

de los artículos al no ser pagado por los lectores recae en las instituciones o sociedades científicas responsables de las revistas, el patrocinio de anunciantes, apoyadores públicos y privados o los autores mediante el cobro de la tarifa de procesamiento del artículo, del inglés article processing charge (APC). Actualmente muchas revistas reconocidas, estan ofreciendo la opción del acceso abierto. Este modelo se conoce como híbrido, en donde ofrecen a los autores la opción de pagar por un artículo que se publicará libremente en internet, conservando los demás artículos accesibles mediante el pago individual o la suscripción a la revista. ${ }^{14}$

Existen revistas de acceso abierto que usan APC con una excelente reputación académica como BioMed Central que almacena cerca de 360 revistas de calidad revisadas por pares en el área de la biomedicina y otras como el mismo SciELO que tiene cerca de 1.000 revistas en todas las áreas del conocimiento de similares características. Los valores de APC pueden variar, incluso eximiendo 
del pago a los autores de países de bajos ingresos según la clasificación del Banco Mundial. ${ }^{13-15}$

En 2015 se publicó en BMC Medicine un estudio acerca de esta temática, encontrando una importante falta de información entre los autores con respecto a este fenómeno. ${ }^{15}$ En este estudio, se identificaron cerca de 1.030 editoriales de las cuales 614 se encontraban en la lista de revistas predadoras de Beall ${ }^{16,17}$ y 416 revistas individuales. Luego del análisis quedaron 966 editoriales y 11873 revistas, se extrajo una muestra representativa de 655 editoriales y 613 revistas, se analizaron variables como el país declarado por las editoriales, la cantidad de artículos publicados en promedio por año en cada revista, la cantidad promedio de revistas publicadas por las editoriales, el país de filiación de los autores y el valor de APC cobrado. Desde el año 2010 hasta el 2014 la cantidad de revistas publicadas por las editoriales depredadoras en todas las temáticas creció de 1.800 a 8.000. El área de conocimiento más común se catalogó como "sin especificar" seguidas por las revistas del área de ingeniería con 97.000 artículos, seguidas de revistas biomédicas con 70.000 artículos en 2014. El país declarado por cada editorial en muchas ocasiones no es verdadero y las direcciones no existen. En la muestra de 655 editoriales, 27\% se localizaban en la India, 17,5 en Norteamérica, $11,6 \%$ en Asia, 8,8\% en Europa, 5,5\% en África y tanto en América del Sur como Australia 1,5\%. El tiempo promedio hasta lograr la publicación en estas revistas fue calculado en 3,6 meses con mediana de 2,7 meses. ${ }^{16}$

Los autores de este estudio, de manera interesante consideran que los autores no son víctimas inocentes y toman una decisión consiente al asumir los riesgos de publicar en estas revistas con la esperanza que se realice siempre un análisis superficial de sus publicaciones ocasionando distorsiones en la evaluación de la idoneidad perpetuando practicas inapropiadas. ${ }^{15}$

En América Latina se tiene un bajo porcentaje de editoriales predadoras relacionado principalmente con la falta de infraestructura en contraste con países de medianos o altos ingresos. Luego de varios esfuerzos para aclarar y determinar el papel de las editoriales y revistas predatorias se concluye que se deben definir criterios de calidad y la acreditación de las revistas con iniciáticas como Directory of Open Access Journals (DOAJ) y la Open Access Scholarly Publishing Associations (OASPA) que realizan la evaluación pertinente en pro de la calidad y credibilidad de las publicaciones realizadas por estas revistas acceso abierto. ${ }^{15-18}$

\section{Publicar a Nivel Regional o Publicar Globalmente: Pros y Contras}

Una revista biomédica de calidad tiene que satisfacer las necesidades tanto del autor como del lector y para esto debe cumplir con funciones como: un adecuado registro, certificación que la investigación se haya llevado a cabo de forma correcta, difusión al público adecuado, acceso a los diferentes tipos de lectores, permanencia de un registro público permanente, y acceso a medios electrónicos. ${ }^{1-5}$

El Factor de Impacto es una herramienta elaborada anualmente por el Instituto para la Información Científica, que tiene como fin medir la influencia, la importancia y el nivel de investigación de las revistas indexadas en la base de citaciones más importante a nivel mundial que pertenece a la Revista de Reportes de Citaciones (Journal Citation Reports). ${ }^{7,8}$ Sin embargo es una herramienta que resulta ser dependiente de la población que lee y tiene acceso a las diferentes revistas indexadas.

Esto ha llevado a que, en el contexto latino, se incremente el interés por el factor de impacto de revistas latinoamericanas, ${ }^{5}$ ya que, si bien existen publicaciones de alta calidad en esta región, la publicación se realiza en revistas extranjeras, principalmente norteamericanas y europeas debido al bajo factor de impacto que poseen revistas del contexto latinoamericano. La implementación de Factor de impacto en muchos de los círculos académicos y de investigación, ha generado un profundo malestar por parte de los investigadores y de las directivas de las diferentes revistas, debido a las limitaciones e innumerables sesgos que tiene como indicador el factor de impacto; una de la principales quejas es la metodología con la que se elabora este indicador, ${ }^{10}$ en donde por ejemplo, la manera en que el Instituto para la Información Científica selecciona las revistas por número de citaciones, resulta ser no representativa en comparación con toda la producción de literatura científica internacional con un sesgo de cobertura que favorece de manera importante a las revistas angloamericanas, ${ }^{3,6,7}$ lo que lleva a que países que no son de habla inglesa tengan menor posibilidad de ser citados y por ende sus revistas reciban puntajes más bajos, lo que para la comunidad científica se ha convertido en una contradicción respecto a los trabajos realizados en países de alta publicación no anglófonos como ocurre con España, seguido de Italia y Francia. ${ }^{7}$

Dentro de los sesgos fundamentales en el factor de impacto se encuentran los múltiples errores que existen al momento de referenciar un artículo, lo que no afecta el valor en el factor de impacto, pero si la calidad del mismo, y debido a esto sólo el $20 \%$ de los artículos publicados son responsable del $80 \%$ de las citaciones, por tanto, las revistas de mayor impacto son aquellas que en sus publicaciones tengan ese $20 \%$ de artículos bien referenciados. ${ }^{7-10}$ El segundo sesgo fundamental se basa en que las revistas muchas veces excluyen dentro del recuento final para el cálculo del factor de impacto aquellos artículos con menor aporte científico, sin embargo, en dado caso que estos artículos sean citados, entrarán en el recuento de citaciones en las que fue mencionada la 
revista; fenómeno que incrementa el numerador y disminuye el denominador, lo que lleva a que el valor del factor de impacto incremente. ${ }^{9,10}$ Un tercer sesgo radica en que se ha demostrado que entre mayor es la longitud del artículo, este tiende a ser citado mayor número de veces, sin que esto garantice que sea de más alta calidad, lo que lleva a una contradicción al momento de calcular el factor de impacto. Un cuarto sesgo se debe al acceso de la literatura electrónica en la red, lo que lleva a que los artículos que se enlistan de primero en los principales buscadores, así como la disponibilidad del texto completo en línea, tengan mayor posibilidad de ser citados. ${ }^{9-11}$

Como consecuencia de lo anterior, las revistas regionales pueden ser subvaloradas, especialmente cuando se publican artículos que hablan de problemas regionales que no tienen impacto mundial, como ocurre con artículos que hablan de problemas de salud pública y atención primaria en salud. Esto lleva en muchos casos a que los autores con el fin de darse a conocer, para optar por becas, programas de especialización y apoyo económico, prefieran investigar poco en situaciones de la población común, para publicar acerca de temas de importancia internacional y considerando primero el envio de artículos en revistas de alto impacto, y dejando como última opción, el publicar en revistas regionales que tengan menor factor de impacto. Este fenómeno puede generar una fuga de la investigación científica en países que manejan idiomas diferentes al inglés, ya que generan una pérdida significativa de los apoyos de investigación.

\section{CONCLUSIÓN}

Cada día son más las opciones para realizar publicaciones científicas lo cual nos obliga a verificar los estándares de calidad de las mismas al momento de usarlas. Los indices bibliométricos como el factor de impacto son útiles para evaluar calidad, pero no deben utilizarse como medida única y pueden complementarse con otro tipo de medidas bibliometricas, en especial en zonas en las cuales existen revistas de bajo impacto. Antes de considerar revistas de acceso abierto de editoriales poco reconocidas, es importante consultar listas de editoriales y revistas predatorias para no caer en un engaño y someter trabajos científicos a una revisión de poca calidad que llevará nuestro artículo a revistas de mala reputación académica a nivel internacional. Revistas generales reconocidas de editoriales de calidad o revistas especializadas de asociaciones científicas de alta calidad académica deben ser la primera opción a considerar independiente de los índices bibliométricos.

\section{REFERENCIAS}

1. Rallison SP. What are Journals for? Ann R Coll Surg Engl. 2015 Mar;97(2):89-91.

2. Sharman A. "Where to publish." Ann R Coll Surg Engl. 2015 July; 97(5): 329-32.

3. Dong P, Loh M, Mondry A. The "impact factor" revisited. Biomed Digit Libr. 2005 Dec 5;2:7.

4. De Vito E L, Algunas consideraciones en torno al uso del Factor de Impacto y de la Bibliometría como herramientade evaluación científica. Revista Americana de Medicina Respiratoria 2006637-45. Disponible en: http://www.redalyc. org/articulo.oa?id=382138365005.

5. Téllez-Zenteno JF, Morales-Buenrostro LE, Estañol B. Análisis del factor de impacto de las revistas científicas latinoamericanas. Rev. méd. Chile [Internet]. 2007 Abr;135(4):480-487. Disponible en: http://www.scielo.cl/scielo.php?script=sci arttext\&pid=S0034-98872007000400010\&lng=es.

6. González G, Castellano M, Valderrama J and Benavent R. Literatura científica de autores españoles sobre análisis de citas y factor de impacto en Biomedicina (1981-2005). Revista española de Documentación Científica, 2008;31(3):344-365.

7. Garfield E. The history and meaning of the journal impact factor. JAMA. 2006 Jan 4;295(1):90-3.

8. Journal Citation Reports. 2015. Available: https://error.incites. thomsonreuters.com/error/Error?DestApp=IC2JCR\&Alias $=\mathrm{IC} 2 \&$ Domain $=$.thomsonreuters.com $\&$ Src $=\mathrm{IP} \&$ Params $=\mathrm{D}$ estApp $\% 3$ DIC2JCR\&RouterURL=https $\% 3 \mathrm{~A} \% 2 \mathrm{~F} \% 2$ Flogin. incites.thomsonreuters.com\%2F\&Error=IPError. Consult in November 05, 2016.

9. Bordons M and Zulueta MA. Evaluación de la actividad científica a través de indicadores bibliométricos, Revista Española de Cardiología, 1999;52(10):790-800.

10. Quindós G. Confundiendo al confuso: reflexiones sobre el factor de impacto, el índice h(irsch), el valor $\mathrm{Q}$ y otros cofactores que influyen en la felicidad del investigador. Rev Iberoam Micol 2009;26:97-102.

11. Omobowale AO, Akanle O, Adeniran AI, Adegboyega K. Peripheral scholarship and the context of foreign paid publishing in Nigeria. Current Sociology. 2014 62;5:666-684.

12. Scientific electronic library online. (SciELO). Chile: 2016. Disponible en: http://analytics.scielo.org/w/publication/ article? collection $=$ chl.

13. World DataBank. Washington, DC (US). 2016. Disponible en: http://databank.worldbank.org/data/home.aspx.

14. Comment L, Nassi-Calò L. Acceso Abierto en América Latina libre de revistas predatorias. SciELO en Perspectiva. 2015. 13; 11:43.

15. Shen C, Björk BC. 'Predatory' open access: a longitudinal study of article volumes and market characteristics. BMC Med. 2015 Oct 1;13:230.

16. Beall J. Predatory journals: Ban predators from the scientific record. Nature. 2016 Jun 15;534(7607):326.

17. Beall J. Predatory publishers are corrupting open access. Nature. 2012 Sep 13;489(7415):179.

18. Beall J. List of publishers. Scholarly Open Access. 2015. Disponible en: http://scholarlyoa.com/publishers/. 